\title{
A INFORMAÇÃO CONTABILÍSTICA NAS DECISÕES FINANCEIRAS DAS PEQUENAS EMPRESAS
}

\section{LEONOR DA C. FERREIRA NUNES}

Professora Assistente no Depto. de Gestão e Economia da Universidade da Beira Interior - Portugal

E-mail: Inunes@fenix.ubi.pt

\section{ZÉLIA Ma DA SILVA SERRASQUEIRO}

Professora Auxiliar do Depto. de Gestão e Economia da Universidade da Beira Interior - Portugal

E-mail: zserrasqueiro@ deimos.ubi.pt

\section{RESUMO}

O presente artigo tem por objectivo analisar a importância da informação contabilística atribuída pelos empresários/gestores das pequenas empresas na tomada de decisão estratégica (que comporta as decisões de investimento, financiamento e distribuição de dividendos) e operacional (relacionada com as decisões de gestão corrente). Assim como, verificar se existem grupos distintos de empresas na importância atribuída à informação contabilística segundo as decisões estratégicas e operacionais e se esses grupos se distinguem entre si, quando a contabilidade é feita na própria empresa ou por um gabinete de contabilidade/consultadoria externa.

Os resultados evidenciam que os empresários/ gestores atribuem uma maior importância à informação contabilística nas decisões de investimento e operacionais do que nas decisões de financiamento e distribuição de dividendos. Constatou-se que, quando a contabilidade é feita na própria empresa, os empresários/gestores atribuem maior importância à informação contabilística tanto nas decisões estratégicas como operacionais.

Palavras-chave: Decisões Financeiras, Informação Contabilística, Pequenas Empresas.

\section{ABSTRACT}

This paper analyzes the role of accounting information for small firms' owners and/or managers for financial (i.e. for making decisions on investment, financing and dividends) as well as operational decision making. Furthermore, this research aims to identify different groups of firms regarding the importance they give to accounting information for strategic and operational decision making, and considering the financial statements elaborated within the company or by an external accounting or consulting firm.

Data were submitted to bivariate and multivariate statistical analyses. The results show that small firms' owners and/or managers confer more importance to accounting information for investment and operational decision making than for financing and dividends decision making. Moreover, the owners and/or managers give more importance to accounting information for strategic and operational decision making when the financial statements are elaborated within the company.

Keywords: Financial Decisions, Accounting Information, Small Firms. 


\section{INTRODUÇÃO}

O empresário/gestor, independentemente da actividade da empresa decide em torno de dois grandes grupos de decisões: estratégicas e operacionais (NEVES, 1996). Enquanto as decisões estratégicas são decisões de médio e longo prazo e englobam as decisões de investimento, financiamento e distribuição de dividendos, as quais têm que ser consideradas de forma interdependente (MCMAHON et al., 1993; NEVES, 1996; NETO, 1997), as decisões operacionais possuem um foco de curto prazo e fazem parte da gestão corrente da empresa.

Esse último conjunto de decisões, as decisões operacionais, segundo McMahon et al., (1993), está relacionado com: decisões do activo circulante, decisões de custo-volume-resultado, decisões do conjunto de produtos e/ou serviços, decisões de fazer/comprar e decisões de preços. Segundo Neves (1996), as decisões operacionais estão relacionadas com a gestão do activo circulante e dos débitos a curto prazo.

As decisões de investimento envolvem todo o processo de identificação, avaliação e selecção das alternativas de aplicação de recursos, conforme identificadas nos activos, enquanto as decisões de financiamento envolvem a definição da natureza dos fundos aplicados, ou seja, a estrutura das fontes de capital procuradas pelas decisões de investimento (SAÁ-REQUEJO, 1996; NEVES, 1996; NETO, 1997). As decisões de financiamento podem reflectir as taxas de retorno exigidas pelos detentores de capital e as oportunidades de investimento centram-se nos retornos esperados (NETO, 1997). Segundo Tibbits (1979), como nas pequenas empresas o tipo de financiamento é o maior constrangimento da decisão de investimento, esses dois tipos de decisões têm que ser analisados em simultâneo.

A inter-relação entre as decisões de financiamento e distribuição de dividendos verifica-se pela opção da empresa em manter um determinado volume de capital próprio financiando os seus investimentos. A decisão de distribuição de dividendos é uma decisão de financiamento mediante capital próprio ao reter dividendos ou de capital de terceiros ao distribuir dividendos (MCMAHON et al., 1993; NETO, 1997).

A tarefa essencial é assegurar, através das decisões de investimento, financiamento e distribuição de dividendos, um retorno suficiente tanto em quantida- de como em tempo, de modo a remunerar os investidores da empresa (MCMAHON e STANGER, 1995).

No presente estudo, procura-se conhecer o papel da informação contabilística na tomada de decisões financeiras das pequenas empresas.

A escolha do tema em questão, além de se basear no interesse pessoal dos autores, decorre também do facto de existirem poucos estudos na área contabilística (TURNER, 1997; TUA PEREDA, 1997), nomeadamente em Portugal, e ao facto dos estudos existentes carecerem de fundamentação empírica (TUA PEREDA, 1997).

A escolha das pequenas empresas recaiu sobre vários factores. Por um lado, porque as pequenas empresas, como refere Bryan e Friedlob (1984), são muito vulneráveis aos problemas derivados de uma insuficiente ou inadequada informação contabilística, sendo necessário o empresário/gestor conhecer os benefícios da utilização da informação contabilística, na tomada de decisão. Por outro lado, porque existem poucos estudos acerca das pequenas empresas (MCMAHON et al., 1993). Por último, as pequenas empresas são de crucial importância para o crescimento económico, tanto em Portugal como no resto da Europa.

$\mathrm{Na}$ Europa, cerca de $98 \%$ das empresas são pequenas empresas, mais de $90 \%$ das empresas têm menos de 10 trabalhadores, e são grandes impulsionadoras do crescimento e do emprego (LANNIELLO, 1999). As razões para a existência destes factores, prendem-se com o facto de as pequenas empresas serem menos vulneráveis às flutuações dos ciclos económicos e de utilizarem mais mão de obra intensiva (LANNIELLO, 1999).

Em Portugal, o papel das pequenas empresas assume também uma importância crucial no desenvolvimento económico. Estas encontram-se presentes em todos os sectores da indústria e serviços, representando, em 1999, cerca de $96,4 \%$ das empresas portuguesas, $50,9 \%$ do emprego e realizam cerca de 34,7\% do volume de negócios nacional (IAPMEI, 2002).

\section{AS DECISÕES FINANCEIRAS}

Enquanto Lusvarghi (1996) se remete para a importância da análise de investimentos nas pequenas empresas, Olivera (1989) refere que a informação contabilística constitui um auxiliar precioso na análise de investimentos. 
Na tomada de decisões de investimento o factor mais relevante para Silva (1999) consiste no custo do capital. No entanto, segundo a investigação de Gonçalves (1997), o conhecimento das disponibilidades financeiras é o elemento mais importante. Kida, Moreno e Smith (2001), por outro lado, referem que a selecção da melhor alternativa para investimento é feita de acordo com o retorno do investimento. Luoma, no seu estudo efectuado em 1967, remete-se para a importância do impacto dessa decisão nos custos futuros e na capacidade financeira da empresa e, também, para o impacto desses efeitos no capital necessário para investimento.

Segundo Holmes e Nicholls (1988), existe uma divergência significativa entre a informação recomendada pelos contabilistas e a utilizada pelos empresários/ gestores na avaliação de projectos de investimento. Por um lado, os contabilistas das pequenas empresas consideram como aspectos mais importantes: o plano empresarial e a elaboração de orçamentos, a contabilidade de gestão, a assessoria a nível das decisões de investimento e financiamento e possíveis implicações dos impostos. Por outro lado, um número significativo de empresários/gestores consideram como informações mais significativas, na análise de investimentos, a pesquisa de mercado e as demonstrações financeiras. De acordo com os mesmos autores, essas diferenças podem reflectir a falta de conhecimentos acerca da informação necessária e disponibilizada pelos contabilistas aos empresários/gestores, ou então, o uso limitado da informação fornecida pelos contabilistas para avaliar as decisões de investimento.

No entanto, o nível de compreensão e uso de técnicas de análise de investimentos é reduzido nas pequenas empresas, assim como a elaboração de orçamentos de investimento, devido à falta de conhecimentos e de recursos humanos em gestão financeira (GRABLOWSKY e BURNS, 1980; MCMAHON et al., 1993; PEEL e WILSON, 1996; ARNOLD E HATZOPOULOS, 2000). Grablowsky e Burns (1980) referem que outra das razões é o custo associado à contratação de um consultor ou contabilista externo familiarizado com essas técnicas. Contudo, vários estudos (TIBBITS, 1979; LEVIN e TRAVIS, 1987) argumentam que as técnicas de análise de investimentos utilizadas pelas grandes empresas não são, necessariamente, importantes para as decisões de investimento das pequenas empresas.
Várias investigações (LUOMA, 1967; FABLONSKY e DIRSMITH, 1979; GRABLOWSKY e BURNS, 1980; PEEL e WILSON, 1996; ARNOLD e HATZOPOULOS, 2000; SERRASQUEIRO e RAPOSO, 2002) analisaram a utilização das técnicas de análise de investimentos nas pequenas empresas. Dos vários estudos efectuados, verifica-se que o período de recuperação é uma técnica muito utilizada nas pequenas empresas, não descurando as outras técnicas de análise de investimentos.

$\mathrm{Na}$ tomada de decisões de financiamento, para além da análise da relação entre o retorno do investimento e o custo do capital, é necessário analisar a relação entre a capacidade de gerar liquidez e os desembolsos exigidos (NETO, 1997). De acordo com Ocaña, Salas e Vallés (1994), para estudar o financiamento das empresas, distinguem-se quatro aspectos principais que são: o nível de endividamento, a composição da dívida, a cobertura da dívida e os custos da dívida, sendo cada um deles analisado mediante um conjunto de rácios elaborados de acordo com a informação contabilística das empresas.

Segundo Menéndez - Requejo (1996), a capacidade da empresa para investir depende em parte da sua capacidade de autofinanciamento e do acesso a financiamento externo à empresa e, por conseguinte, das condições em que esse se obtém. Nas pequenas empresas, o endividamento é, geralmente, de curto praZo (OSTERYOUNG; CONSTAND; NAST, 1992; OCAÑA; SALAS; VALLÉS, 1994) e as instituições bancárias, segundo Gastón e Jarne (1995), são a fonte de financiamento mais importante. Os gestores bancários exigem informações contabilísticas para analisar o desempenho passado da empresa, de modo a assegurar os melhores termos e condições de negociação do empréstimo (OLIVERA e MARTIN, 1993; CORMIER e MAGNAN, 1996; KWOK, 2002). No entanto, devido ao maior risco de falência associado ao investimento, as pequenas empresas deparam-se com custos de capital mais elevados (ANG, 1991; HOLMES e KENT, 1991) constituindo uma dificuldade para essas empresas no recurso ao capital alheio (IAPMEI/DPDPME, 1995).

Na obtenção de financiamento, a existência de um contrato jurídico que obrigue a empresa a pagar montantes fixos numa data pré-estabelecida, pode originar que os empresários/gestores se deparem com dificuldades de tesouraria, comprometendo a situação financeira da empresa (DONALDSON, 1962). 
Gonçalves (1997), no seu estudo, concluiu que as informações consideradas mais importantes para a tomada de decisões de financiamento são, respectivamente, o impacto na estrutura financeira, a necessidade de equilibrar a tesouraria e os custos associados ao endividamento.

De acordo com Santos (1994), as informações contabilísticas podem servir para equacionar a política de distribuição de dividendos, tendo em conta os objectivos dos investidores e da empresa. Contudo, a retenção de lucros é considerada uma fonte importante de financiamento nas pequenas empresas (GRABLOWSKY e BURNS, 1980; LECORNU et al., 1996; JESUS; ROCHA; VIANA, 2002), à medida que pode evitar o recurso a capital alheio (MENÉNDEZ REQUEJO, 1996), limitando, desse modo, o risco financeiro da empresa e a probabilidade de falência (JENSEN; SOLBERG; ZORN, 1992).

Hall e Young citado em McMahon et al. (1993), referem que a falência, na maioria das empresas, é devida a problemas de gestão operacional. Vários estudos remetem para a importância da análise periódica: das dívidas a pagar e a receber (BERGSMAN, 1992; GONÇALVES, 1997), das existências em armazém (BERGSMAN, 1992; GADENNE, 1998), dos níveis de disponibilidades (GADENNE, 1998) e do crédito concedido (CHITTENDEN e BRAGG, 1997; PIKE e CHENG, 2001).

Robinson e Wilson (1993) referem que os empresários/gestores das pequenas empresas, devido à dificuldade em obter financiamento externo, colocam uma maior ênfase no financiamento dos proprietários e na gestão do activo circulante (como é o caso do crédito dos fornecedores).

\section{OBJECTIVOS E AMOSTRA DE INVESTIGAÇÃO}

Em face da exposição anterior, relacionada com as decisões financeiras, o objectivo geral do presente trabalho consiste em averiguar o grau de importância atribuído pelos empresários/gestores à informação contabilística nas decisões de investimento, financiamento, distribuição de dividendos e decisões operacionais. Além disso, o presente trabalho tem ainda por objectivo testar se existem grupos distintos de empresas na importância atribuída à informação contabilística na tomada de decisão estratégica e operacional e se essa importância varia, consoante a contabilidade seja feita interna ou externamente à empresa.

Com o intuito de atingir os objectivos definidos elaborou-se um questionário, que foi enviado por correio em Março de 2003, às empresas portuguesas com menos de 50 trabalhadores do distrito de Castelo Branco. A escolha do distrito de Castelo Branco incidiu sobre o facto de nele se inserir a Universidade da Beira Interior, pois existe desta forma uma maior disponibilidade dos empresários/gestores para colaborar em investigações desenvolvidas pelos membros da universidade. Por outro lado, a homogeneidade encontrada em termos da responsabilidade pela área contabilístico/financeira, que é na maioria das pequenas empresas o proprietário, dão relevância à incidência apenas sobre um distrito de Portugal continental.

No primeiro grupo do questionário, obtiveram-se informações acerca da empresa e do empresário/ gestor, assim como se a contabilidade era feita na própria empresa ou externamente. Num outro grupo, procurou-se verificar a importância da informação contabilística nas decisões financeiras, através de uma escala de Likert de 1- nada importante a 5 muito importante.

Após terminado o período de respostas obtiveramse 66 questionários devidamente preenchidos, dos quais resultou uma taxa de resposta de cerca de $22 \%$. A análise dos resultados foi feita no programa informático SPSS (Statistical Package for the Social Sciences), em que o tipo de tratamento estatístico aplicado foi função do tipo de questão envolvida, nomeadamente da escala de medida das variáveis e do propósito do tratamento.

\section{ANÁLISE DOS RESULTADOS}

\subsection{A importância da informação contabilística nas decisões financeiras}

De acordo com o estudo de Gabas - Trigo et al., (1996), as demonstrações financeiras, segundo os utilizadores internos, são úteis, respectivamente, para as decisões de distribuição de dividendos, conhecimento geral da empresa, decisões de financiamento e, por último, para as decisões de investimento.

Na presente investigação, os inquiridos conside- 
ram a informação contabilística, em média, mais importante nas decisões de investimento (média de 4,2), de seguida nas decisões operacionais (média de 4,07). Esse resultado pode estar relacionado com o facto de a gestão das pequenas empresas ser baseada numa perspectiva de curto prazo (RICE e HAMILTON, 1979; GRABLOWSKY e BURNS, 1980; LUSVARGHI, 1996) e por isso, na maioria dos casos essas empresas preferem investimentos de menor duração (LE PERS, 1979) e, consequentemente, empréstimos de curto prazo (OSTERYOUNG; CONSTAND; NAST, 1992; OCAÑA; SALAS; VALLÉS, 1994).

Na decisão de financiamento, os empresários/ gestores atribuem uma média de 3,97 e nas decisões de distribuição de dividendos consideram a informação contabilística menos importante, quando comparada com as variáveis anteriores, atribuindo uma média de 3,43. Esse último resultado, pode derivar de, na maioria das pequenas empresas, os proprietários, ao ocuparem funções de gestão e a propriedade estar concentrada num reduzido número de pessoas, procederem a uma menor distribuição de dividendos, com o objectivo de evitarem o recurso ao financiamento externo à empresa (JENSEN; SOLBERG; ZORN, 1992; REQUEJO, 1996; JESUS; ROCHA; VIANA, 2002). Contudo, nas pequenas empresas, devido à flexibilidade da gestão e à ausência de responsabilidade limitada, os seus proprietários, apesar de não receberem dividendos, recebem-nos indirectamente através, por exemplo, do uso de activos da empresa para fins pessoais (ANG, 1991).

Com o propósito de analisar o tipo de informações que são consideradas mais importantes pelos empresários/gestores nas decisões de investimento, financiamento e distribuição de resultados, assim como nas decisões operacionais, eles foram questionados acerca do grau de importância da informação contabilística, nesse tipo de decisões, segundo determinados itens, que se apresentam no quadro seguinte.

\begin{tabular}{|c|c|c|c|}
\hline VARIÁVEIS & № de empresas & Média & Desvio padrão \\
\hline \multicolumn{4}{|l|}{$\begin{array}{l}\text { Nas decisões estratégicas } \\
\mathrm{Na} \text { decisão de investimento }\end{array}$} \\
\hline Custos associados ao investimento & 65 & 4,11 & 0,986 \\
\hline Rendibilidade esperada do investimento & 65 & 4,05 & 1,052 \\
\hline Período de recuperação do investimento & 65 & 4,03 & 1,089 \\
\hline Capacidade financeira da empresa & 66 & 4,39 & 0,839 \\
\hline Fluxo de caixa & 65 & 4,02 & 0,910 \\
\hline \multicolumn{4}{|l|}{ Na decisão de financiamento } \\
\hline Impacto na estrutura financeira da empresa & 64 & 4,17 & 1,017 \\
\hline Custos associados ao financiamento & 64 & 4,03 & 0,942 \\
\hline Necessidade de equilibrar a tesouraria & 65 & 3,94 & 1,144 \\
\hline Necessidade de financiar investimentos & 64 & 3,95 & 1,045 \\
\hline $\begin{array}{l}\text { Capacidade para fazer face aos encargos } \\
\text { correntes dos empréstimos }\end{array}$ & 65 & 4,12 & 1,038 \\
\hline \multicolumn{4}{|l|}{ Na decisão de distribuição de dividendos } \\
\hline O montante dos lucros já retidos & 62 & 3,66 & 1,055 \\
\hline Taxa de tributação dos dividendos & 58 & 3,57 & 1,126 \\
\hline Capacidade financeira da empresa & 61 & 4,13 & 0,991 \\
\hline \multicolumn{4}{|l|}{ Nas decisões operacionais } \\
\hline Prazos médios de recebimento e pagamento & 66 & 4,06 & 0,990 \\
\hline Listagem da antiguidade de saldos devedores e credores & 65 & 4,11 & 0,986 \\
\hline Nível de existências em armazém & 65 & 3,69 & 1,236 \\
\hline Margens de lucro praticadas & 65 & 3,86 & 1,044 \\
\hline Volume das disponibilidades & 66 & 3,92 & 0,966 \\
\hline Análise dos indicadores financeiros da empresa & 66 & 4,08 & 0,933 \\
\hline $\begin{array}{l}\text { Comparação dos indicadores financeiros da empresa } \\
\text { com os da concorrência }\end{array}$ & 66 & 3,52 & 1,256 \\
\hline Aplicação dos recursos de tesouraria & 66 & 3,65 & 1,074 \\
\hline
\end{tabular}

\section{Quadro 1 - Importância da informação contabilística nas decisões estratégicas e operacionais}

Os valores apresentados foram calculados com base numa escala numérica de 5 pontos (1 - nada importante a 5 - muito importante). 
Da análise do quadro anterior, constata-se que os empresários/gestores, na tomada de decisões de investimento, consideram a informação contabilística importante, para analisar, respectivamente, a capacidade financeira da empresa (média de 4,39), os custos associados ao investimento (média de 4,11), a rendibilidade esperada do investimento (média de 4,05), o período de recuperação do investimento (média de 4,03) e, por último, para determinar o fluxo de caixa (média de 4,02). Verifica-se, assim, que os empresários/gestores atribuem, em média, um grau bastante importante a todos os itens referidos anteriormente.

Também na tomada de decisões operacionais, como referido anteriormente, os empresários/ gestores atribuem, em média, bastante importância às informações proporcionadas pela contabilidade. A comparação dos indicadores financeiros da empresa com os da indústria assume em média menor importância (Quadro 1). A informação contabilística com maior importância na tomada de decisões operacionais corresponde à listagem da antiguidade dos saldos devedores e credores (média de 4,11, conforme Quadro 1), enquanto, no estudo de Gonçalves (1997), se tratava da informação menos importante.

Conforme a análise do Quadro 1, os inquiridos consideram que a informação contabilística na tomada de decisões de financiamento é, em média, bastante importante para determinar, respectivamente: o impacto na estrutura financeira da empresa, a capacidade de a empresa fazer face aos encargos correntes dos empréstimos obtidos, os custos associados ao financiamento, a eventual necessidade de financiar potenciais investimentos e, por fim, a necessidade de a empresa equilibrar a tesouraria. No estudo de Gonçalves (1997), a informação contabilística considerada mais importante foi, também, o impacto na estrutura financeira da empresa.

Desse modo, os resultados sugerem que as pequenas empresas se preocupem com a estrutura financeira, remetendo-nos para o estudo de Ang (1991), segundo o qual essas empresas estão expostas a um maior risco financeiro e por isso, na tomada de decisões de financiamento, têm de considerar o impacto na sua estrutura financeira.
A análise da capacidade financeira da empresa é o factor considerado mais importante na decisão de distribuição de dividendos. $O$ factor com menor importância consiste na taxa de tributação dos dividendos, com uma média de 3,57. Esses resultados, em conjunto, sugerem que nas pequenas empresas as decisões de distribuição de dividendos estão relacionadas com as decisões de financiamento, mostrando uma preferência pelo autofinanciamento, de acordo com os estudos de Myers e Majluf (1984), Barton e Matthews (1989), Saá-Requejo (1996), Hamilton e Fox (1998) e Watson e Wilson (2002).

\subsection{Grupos distintos de empresas na importância atribuída à informação contabilística}

Após a análise da importância atribuída à informação contabilística na tomada das decisões estratégica e operacional, procurou-se testar se existiam grupos distintos de empresas, segundo a importância atribuída à informação contabilística nas decisões estratégicas e operacionais, procedendo-se a uma análise exploratória de cluster.

Segundo Hair et al. (1998) a análise de clusteré uma técnica exploratória, que agrupa observações, de forma a maximizar a variância entre os grupos e minimizar a variância dentro dos grupos. Na análise dos clusters, de entre as várias medidas de distâncias possíveis, existe a medida do Quadrado da Distância Euclideana, que consiste na raiz quadrada do somatório dos quadrados das diferenças entre valores de dois casos ( $\mathrm{i}$ e j) para todas as variáveis (REIS, 1991). Optou-se por essa medida por ser a mais utilizada em variáveis quantitativas (PESTANA e GAGEIRO, 2003) e no método Ward (MALHOTRA, 1993; HAIR et al., 1998). O critério de agregação ou desagregação dos casos utilizados foi o método Ward, que pretende optimizar a variância mínima dentro dos grupos. Esse método foi adoptado por ser um dos métodos que melhores resultados apresenta (HAIR et al., 1998).

Uma das questões essenciais que se coloca na análise de cluster, relaciona-se com o número de clusters a formar. Essa decisão é considerada difícil, uma vez que não existe um critério estatístico que permita determinar com precisão o número "ideal" de clusters (HAIR et al., 1998). Desse modo, a selecção 
do número de clusters a formar baseou-se na observação do dendograma e do coeficiente de fusão, pois são os métodos mais aconselhados segundo Hair, et al. (1998) e Pestana e Gageiro (2003).

A análise de cluster foi realizada a partir das variáveis originais, relativas à importância da informação contabilística nas decisões de investimento, financiamento, distribuição de dividendos e decisões operacionais. De acordo com a análise do coeficiente de fusão e do dendograma, optou-se pela formação de dois clusters, cujas dimensões são: o cluster 1 com 34 empresas e o cluster 2 com 20 empresas.

Com o objectivo de testar eventuais diferenças significativas nas médias dos clusters, para cada uma das variáveis utilizadas, foi conduzida uma análise da variância One-Way Anova, pois essa procura testar a hipótese nula das médias de os diferentes grupos serem iguais. Como variáveis independentes consideraram-se os clusters obtidos e como variáveis dependentes as variáveis originais utilizadas para formar os clusters. Uma vez que os pressupostos do teste One-Way Anova não foram cumpridos, aplicouse o teste não paramétrico Kruskall Wallis para verificar se a ausência de pressupostos não tinha perturbado os resultados. O teste mostrou a existência de diferenças significativas nos clusters segundo as variáveis dependentes.

$\mathrm{Na}$ interpretação dos clusters, tem especial importância a média que cada cluster assume em cada uma das variáveis dependentes. No Quadro 2 pode observar-se o número de clusters formados e ainda a média e o desvio padrão, quando cada variável dependente é cruzada com cada cluster. Pela visualização dos valores médios, verifica-se que as empresas que pertencem ao cluster 1 atribuem uma importância superior à informação contabilística nas decisões estratégicas e operacionais em relação às pertencentes ao cluster 2 .

\begin{tabular}{|c|c|c|c|c|}
\hline \multirow[t]{2}{*}{ VARIÁVEIS } & \multicolumn{2}{|c|}{ Cluster $1(\mathrm{~N}=34)$} & \multicolumn{2}{|c|}{ Cluster $2(\mathrm{~N}=20)$} \\
\hline & Média & Desvio padrão & Média & Desvio padrão \\
\hline \multicolumn{5}{|l|}{ Nas decisões estratégicas } \\
\hline \multicolumn{5}{|l|}{ Decisões de investimento } \\
\hline Custos associados ao investimento & 4,5 & 0,564 & 3,40 & 1,231 \\
\hline Rendibilidade esperada do investimento & 4,47 & 0,662 & 3,30 & 1,218 \\
\hline Período de recuperação do investimento & 4,59 & 0,557 & 3,10 & 1,165 \\
\hline Capacidade financeira da empresa & 4,79 & 0,410 & 3,80 & 1,056 \\
\hline Fluxo de caixa & 4,53 & 0,507 & 4,02 & 0,961 \\
\hline \multicolumn{5}{|l|}{ Decisões de financiamento } \\
\hline Impacto na estrutura financeira da empresa & 4,47 & 0,861 & 3,55 & 1,146 \\
\hline Custos associados ao financiamento & 4,29 & 0,836 & 3,50 & 1,051 \\
\hline Necessidade de equilibrar a tesouraria & 4,41 & 0,836 & 3,10 & 1,165 \\
\hline Necessidade de financiar investimentos & 4,41 & 0,821 & 3,05 & 0,945 \\
\hline \multicolumn{5}{|l|}{ Capacidade para fazer face aos encargos } \\
\hline correntes dos empréstimos & 4,53 & 0,825 & 3,35 & 1,089 \\
\hline \multicolumn{5}{|l|}{ Decisões de distribuição de dividendos } \\
\hline O montante dos lucros já retidos & 4,12 & 0,808 & 3,00 & 0,918 \\
\hline Taxa de tributação dos dividendos & 4,06 & 0,776 & 2,80 & 1,105 \\
\hline Capacidade financeira da empresa & 4,47 & 0,706 & 3,55 & 1,191 \\
\hline \multicolumn{5}{|l|}{ Nas decisões operacionais } \\
\hline Prazos médios de recebimento e pagamento & 4,53 & 0,615 & 3,30 & 0,979 \\
\hline Listagem da antiguidade de saldos devedores e credores & 4,53 & 0,507 & 3,35 & 1,226 \\
\hline Nível de existências em armazém & 4,38 & 0,697 & 2,65 & 1,309 \\
\hline Margens de lucro praticadas & 4,44 & 0,613 & 2,95 & 0,887 \\
\hline Volume das disponibilidades & 4,53 & 0,563 & 3,05 & 0,887 \\
\hline Análise dos indicadores financeiros da empresa & 4,62 & 0,551 & 3,25 & 0,851 \\
\hline $\begin{array}{l}\text { Comparação dos indicadores financeiros, } \\
\text { com os da concorrência }\end{array}$ & 4,24 & 0,855 & 2,40 & 1,095 \\
\hline Aplicação dos recursos de tesouraria & 4,32 & 0,589 & 2,65 & 0,875 \\
\hline
\end{tabular}

Quadro 2 - Média dos clusters nas variáveis dependentes 
Com o objectivo de verificar se os grupos apresentavam diferenças significativas, quando a contabilidade é elaborada interna ou externamente, aplicouse o teste t. Mas, na ausência dos pressupostos da normalidade e da homocedasticidade, procedeu-se à aplicação do teste não paramétrico Mann-Whitney, que detectou a existência de diferenças significativas, com um grau de significância estatística de 0,1.

Em conformidade com o Quadro 3, verifica-se que, em $64,7 \%$ das empresas pertencentes ao cluster 1 (as que atribuem maior importância à informação contabilística nas decisões estratégicas e operacionais), predomina a contabilidade feita na própria empresa, enquanto no cluster 2 , em $60 \%$ das empresas pertencentes a este grupo, a contabilidade é feita externamente.

\begin{tabular}{l|cc|c}
\multirow{2}{*}{ CLUSTERS } & \multicolumn{2}{|c|}{$\begin{array}{c}\text { Elaboração da contabilidade } \\
\text { interna/externamente }\end{array}$} & \multirow{2}{*}{ Total } \\
\cline { 2 - 3 } & Internamente & Externamente & \\
\hline Cluster 1 & 22 & 12 & 34 \\
& $64,7 \%$ & $35,3 \%$ & $100,0 \%$ \\
\hline Cluster 2 & 8 & 12 & 20 \\
& $40,0 \%$ & $60,0 \%$ & $100,0 \%$ \\
\hline Total & 30 & 24 & 54 \\
& $55,6 \%$ & $44,4 \%$ & $100,0 \%$ \\
\hline
\end{tabular}

Quadro 3 - Caracterização dos clusters com base na elaboração da contabilidade interna/externamente

Esse último resultado pode dever-se ao facto de, quando a contabilidade da empresa é feita externamente, o nível de formação acadêmica do empresário/gestor predominante, no presente estudo, é básico/secundário, e por isso podem apresentar dificuldades na análise e interpretação das várias demonstrações financeiras, não considerando importante a informação contabilística na tomada de decisões estratégicas e operacionais.

Os resultados evidenciam que existem empresários/gestores (pertencentes ao cluster 1 e com maior formação acadêmica) que consideram importante a utilização da informação contabilística na tomada de decisões estratégicas e operacionais. Constatou-se, também, que existem grupos distintos de empresas segundo a importância atribuída à informação contabilística nas decisões estratégicas e operacionais e que esses grupos se distinguem entre si, quando a contabilidade é feita interna ou externamente.

\section{CONCLUSÕES}

Os empresários/gestores, independentemente da actividade da empresa, decidem em torno de dois grandes grupos de decisões: decisões estratégicas, que englobam as decisões de investimento, financiamento e distribuição de dividendos, e decisões operacionais. No presente estudo, os empresários/ gestores consideram que as informações contabilísticas assumem maior importância na tomada de decisões de investimento e decisões operacionais, enquanto nas decisões de financiamento e de distribuição de dividendos a informação contabilística tem uma importância menor.

$\mathrm{Na}$ tomada de decisões de investimento e distribuição de dividendos, os empresários/gestores consideram que a informação contabilística com maior importância é a que possibilita analisar a capacidade financeira da empresa, enquanto na tomada de decisões de financiamento é a que permite avaliar o impacto dessa decisão na estrutura financeira da empresa. Na tomada de decisões operacionais, a informação contabilística tida como mais relevante, corresponde à listagem da antiguidade dos saldos devedores e credores, enquanto a informação menos importante respeita à comparação dos indicadores financeiros da empresa, com os publicados para o mesmo sector de actividade. Contudo, importa salientar que os empresários/ gestores, quando questionados acerca do grau de importância da informação contabilística nas decisões estratégicas e operacionais, segundo determinados itens, atribuem, em média, um grau bastante importante a todos esses itens.

Os resultados permitem verificar que são os empresários/gestores que elaboram a contabilidade internamente que maior importância atribuem à informação contabilística, tanto nas decisões estratégicas como operacionais. Isso poderá indicar que, quando a contabilidade é feita externamente, a principal finalidade da sua elaboração pode ser para propósitos fiscais e legais.

No presente trabalho, verificou-se, ainda, que o nível de formação do empresário/gestor tem influência 
na elaboração da contabilidade, interna ou externamente. Constatou-se que, quando a contabilidade é feita externamente, o nível de formação do empresário/gestor predominante é o ensino básico e secundário. Dessa forma, o empresário/gestor pode apresentar dificuldades na análise e interpretação das demonstrações financeiras, não considerando a informação contabilística um factor importante na tomada de decisões estratégicas e operacionais, necessitando, conseqüentemente, de aconselhamento. Nessa situação, o contabilista pode desempenhar um papel importante de aconselhamento junto do empresário/gestor.

Por último importa salientar que as conclusões resultaram do estudo na sua globalidade, pelo que as tendências gerais podem não ser verdadeiras para um caso particular. Importa também referir que de acordo com a literatura existente há um conjunto considerável de razões, que levam a acreditar que a gestão financeira das pequenas empresas é qualitativa e quantitativamente diferente das grandes empresas (TIBBITS, 1979; LEVIN e TRAVIS, 1987; ANG, 1991; MCMAHON et al., 1993; LECORNU et al., 1996). Pois, enquanto que na maioria das pequenas empresas o responsável pela gestão é o proprietário, nas grandes empresas é o gestor. Deste modo os resultados obtidos podem não se verificar neste último conjunto de empresas. Desta forma uma linha de investigação futura seria confrontar os resultados deste estudo com os resultados obtidos por um estudo idêntico, mas efectuado sobre empresas de grande dimensão.

\section{BIBLIOGRAFIAS}

ANG, J. S. Small Business Uniqueness and the Theory of Financial Management, The Journal of Small Business Finance, v. $1, \mathrm{n}^{\circ} 1$, p. 1-13, 1991.

ARNOLD, G. C. e HATZOPOULOS, P. D. The Theory-Practice Gap in Capital Budgeting: Evidence from the United Kingdom, Journal of Business Finance \& Accounting, v. 27, no 5 e 6, June/ July, p. 603-626, 2000.

BARTON, S. L. e MATTHEWS, C. H. Small Firm Financing: Implications from a Strategic Management perspective, Journal of Small Business Management, January, v. 27, no 1, p. 1-7, 1989.

BERGSMAN, S. Accounting for Small Businesses, Black Enterprise, v. 23, № 4, November, Nova lorque, 1992.

BRYAN, E. L. e FRIEDLOB, G. T. Financial Management and Capital Formation in Small Business, Journal of Small Business Management, Julho, p. 73-75, 1984.

CHITTENDEN, F. e BRAGG, R. Trade Credit, Cash-flow and SMEs in the UK, Germany and France, International Small Business Journal, v. 16, oㅡ 1, p. 22-35, 1997.

CROMIER, D. e MAGNAN, M. Decisions, decisions - What motivates managers to chose certain policies and methods?, CA Magazine, September, p. 38-41, 1996.

FABLONSKY, S. F. e DIRSMITH, M. W. L'information financière externe et les prises de décision dans l'entreprise, La Revue du Financier, décembre, № 6, p. 9-12, 1979.

GABÁS - TRIGO, F; ABADÍA, J. M. M; PÉREZ-GRUESO, A. J. B. e JARNE, J. I. J. Análisis de la demanda de información financiera en la coyuntura actual, Revista Española de Financiación y Contabilidad, v. XXV, № 86, enero-marzo, p. 103-137, 1996.

GADENNE, David, Critical success factors for small business: An inter-industry comparison, International Small Business Journal, v. 17, № 1, p. 36-51, 1998.

GASTÓN, S. C. e JARNE, J. I. J. La Información Financiera en le Contexto Internacional: Análisis Descriptivo, Revista Española de Financiación y Contabilidad, v. XXIV, № 85, Abril/Junio, p. 937-968, 1995.
GONÇALVES, M. F. A importância da Contabilidade para os gestores das PME, Dissertação de mestrado, ISCET, Lisboa, 1997.

GRABLOWSKY, B. J. e BURNS, W. L. The Application of Capital Allocation Techniques by Small Business, Journal of Small Business, v. 18, № 3, p. 50-58, 1980.

HAIR, J. F; ANDERSON, R. E; TATHAM, R. L. e BLACK, W. C. (1998), Multivariate Data Analysis, (ed.) Prentice Hall, Inc., Fifth edition, 1998.

HAMILTON, R. T. e FOX, M. A. The Financing preferences of small firm owners, International Journal of Entrepreneurial Behavior \& Research, v. 4, № 3, p. 239-248, 1998.

HOLMES, S. e KENT,P. An empirical analysis of the financial structure of small and large Australian manufacturing enterprises, The Journal of Small Business Finance, v. 1, № 2, p. 141-154, 1991.

. e NICHOLLS, D. An Analysis of the Use of Accounting Information by Australian Small Business, Journal of Small Business Management, v. 26, № 2, p. 57-68, 1988.

IAPMEI - Instituto de Apoio às Pequenas e Médias Empresas e ao Investimento; Estrutura empresarial Nacional, in <http:// www.iapmei.pt>, 2002.

IAPMEI/DPDPME. O financiamento como factor chave de sucesso para o desenvolvimento empresarial, Pequena e Média Empresa, Instituto de Apoio às Pequenas e Médias Empresas e ao Investimento, $\mathrm{n}$ ㅇ 7-III série trimestral, 1995.

JENSEN, M.C; SOLBERG, D.P. e ZORN, T.S. Simultaneous determination of insider ownership, debt and dividend policies, Journal of Financial and Quantitative Analysis, v. 27, № 2, p. 247-263, 1992.

JESUS, J. R; ROCHA, L. M. e VIANA, R. C; Avaliação de PME's e Gestão de Risco, Revista de Contabilidade e Comércio, Janeiro, no 230, v. LVIII, p. 378-401, 2002.

KIDA, T. E; MORENO, K. K. e SMITH, J. F. The Influence of Affect on Managers' Capital-Budgeting Decisions, Contemporary Accounting Research, v. 18, № 3, p. 477-494, 2001. 
KWOK, $\mathrm{H}$. The effect of cash flow statement format on lenders' decisions, he International Journal of Accounting, № 37, p. 347362, 2002.

LANNIELLO, F. O papel da Pequena Empresa na EU, Edições GEPE - Gabinete de Estudos e Prospectiva Económica do Ministério da Economia, Setembro, Lisboa, 1999.

LE PERS, P. Les responsables financiers et comptables des entreprises, La Revue du Financier, décembre, n6, p. 30-53, 1979.

LECORNU, M. R; MCMAHON, R. G. P; FORSAITH, D. e STANGER, A. M.J. The small enterprise financial objective function, Journal of Small Business Management,v. 34, № 3, p. $1-13,1996$

LEVIN, R. I. e TRAVIS, V. R. Small company finance: what the books don't say, Harvard Business Review,v. 65, № 6, p. 3032, 1987.

LUOMA, G. A. Accounting Information in Managerial DecisionMaking for Small and Medium Manufacturers, Research Monograph 2, National Association of Accountants, December, 1967.

LUSVARGHI,V. The Role of the Management Accountant in Small and Medium-sized Companies, in <www.ifac.org.>, 1996.

MALHOTRA, N. Marketing Research: An Applied Orientation, Prentice-Hall International Inc, 1993.

MCMAHON, R. G. P; HOLMES, S; HUTCHINSON, P. J. e FORSAITH, D. M. Small enterprise financial management Theory \& Practice, Harcourt Brace \& Company, Australia, 1993.

. e STANGER, A. M.J. Understanding the small enterprise financial objective function, Entrepreneurship Theory and Practice, v. 19, № 4, p. 21-38, 1995.

MYERS, S. C. e MAJLUF, N. Corporate Financing and Investment Decisions When Firms Have Information That Investors Do Not Have, Journal of Finance Economics, v. 13, p. 187-221, 1984.

NETO, A. A. A Dinâmica das decisões Financeiras, Caderno de Estudos, São Paulo, FIFECAPI, v. 16, Julho/Dezembro, p. 9-25, 1997.

NEVES, J. C. Análise Financeira -Vol. I - Técnicas Fundamentais, $12^{\mathrm{a}}$ ed., Texto, 1996.

OCAÑA, C; SALAS, V. e VALLÉS, J. Un análisis empírico de la financiación de la pequeña y mediana empresa manufacturera española: 1983-1989, Moneda y crédito, ㄲo 1999, 1994.

OLIVEIRA, Camilo C. "Derecho de Contabilidad: Finalidad y Fidelidad de las Cuentas Anuales", Revista Española de Financiación y Contabilidad, v. XVIII, № 61, Out/Dec, p. 903913, 1989.

OLIVERA, H. E. e MARTIN,C. Accounting problems Encountered in Small Business Failures, Southwest Small Business Institute Association - SSBIA, 1993.
OSTERYOUNG, J; CONSTAND, R. L. e NAST, D. Financial Ratios in Large Public and Small Private Firms, Journal of Small Business Management, Julho, p. 35-46, 1992.

PEEL, M. J. e WILSON, N. Working Capital and Financial Management Practices in the Small Firm Sector, International Small Business Journal, v. 14, no2, p. 52-68, 1996.

PESTANA, M. H. e GAGEIRO, J. N. Análise de dados para ciências sociais - A complementaridade do SPSS, Edições Sílabo, 3ํㅡㄹ., Lisboa, 2003.

PIKE, R. e CHENG, N. S. Credit Management: An Examination of Policy Choices, Practices and Late Payment in UK Companies, Journal of Business Finance \& Accounting, v. 28, № 7 e 8, Sept./Out., p. 1013-1042, 2001.

MENÉNDEZ - REQUEJO, S. Interdependencia de las Decisiones Financieras en las Empresas Españolas, Revista Española de Financiación y Contabilidad, v. XXV, № 87, Abril-Junio, p. 315342,1996

RICE, G. H. e HAMILTON, R. E. Decision Theory And The Small Businessman, American Journal of Small Business, v. IV, № 1, p. 1-9, 1979.

ROBINSON, A. e WILSON,N. An Empirical Comparison of Cooperative and Private Small Firms in Britain, International Business Review, v. 2, ํㅡ 3, p. 281-296, 1993.

SAÁ-REQUEJO, J. Financing Decisions: Lessons from the Spanish Experience, Financial Management, v. 25, № 3, Autumn, p. 44-56, 1996.

SANTOS, N. Aspectos relacionados com o lucro passível de distribuição e a manutenção do capital da em presa, Cadernos de Estudos, FIPECAFI, S. Paulo, 1994.

SERRASQUEIRO, Z. M. S. e RAPOSO, M. L. B. Capital Budgeting Practices: An Empirical Approach to Portuguese SMEs, Conference Proceedings, RENT XVI- Research Entrepreneurship and Small Business, $16^{\text {th }}$ Workshop, November 21-22, Barcelona, Spain, 2002.

SILVA, P. P. Técnicas de Análise de Investimentos - do VAL às Opções Reais"; Boletim de Ciências Económicas, v. XLII, Universidade de Coimbra, Coimbra, 1999.

TIBBITS, G. E. Small Business Management: A Normative Approach, MSU Business Topics, v. 27, № 4, p. 173-187, 1979.

TUA PEREDA, J. Lecturas de Teoría e Investigación Contable, Centro interamericano jurídico-financiero, CIJUF, Medellín, Colombia, 1997

TURNER, R. Management accounting and SMEs: A question of style?, Management Accounting, Vol. 75, № 7, p. 24-27, 1997.

WATSON, R. e WILSON,N. Small and Medium Sized Enterprise Financing: A Note on Some of the Empirical Implications of a Pecking Order, Journal of Business Finance \& Accounting, v. 29, № 3 e 4, April/May, p. 557-578, 2002.

\section{NOTA:}

Endereço das autoras:

Universidade da Beira Interior

Departamento de Gestão e Economia

Estrada do Sineiro

6200-209 Covilhã - Portugal 